\section{(1)}

CrossMark

\title{
Does early onset asthma increase childhood obesity risk? A pooled analysis of 16 European cohorts
}

\author{
Zuelma A. Contreras ${ }^{1}$, Zhanghua Chen ${ }^{1}$, Theano Roumeliotaki², \\ Isabella Annesi-Maesano ${ }^{3}$, Nour Baïz ${ }^{3}$, Andrea von Berg ${ }^{4}$, Anna Bergström ${ }^{5,6}$, \\ Sarah Crozier ${ }^{7}$, Liesbeth Duijts $\mathbb{1}^{8,9,10}$, Sandra Ekström ${ }^{5}$, Esben Eller ${ }^{11}$, \\ Maria P. Fantini ${ }^{12}$, Henrik Fomsgaard Kjaer ${ }^{11}$, Francesco Forastiere ${ }^{13}$, \\ Beatrix Gerhard ${ }^{14}$, Davide Gori ${ }^{12}$, Margreet W. Harskamp-van Ginkel (15 ${ }^{15}$ \\ Joachim Heinrich ${ }^{16,17}$, Carmen Iñiguez ${ }^{18,19,20}$, Hazel Inskip ${ }^{7,21}$, Thomas Keil ${ }^{22}$, \\ Manolis Kogevinas ${ }^{20,23,24,25}$, Susanne Lau ${ }^{26}$, Irina Lehmann ${ }^{27}$, Dieter Maier ${ }^{14}$, \\ Evelien R. van Meel ${ }^{8,9,28}$, Monique Mommers ${ }^{29}$, Mario Murcia ${ }^{19,20}$, \\ Daniela Porta (10 ${ }^{13}$, Henriëtte A. Smit ${ }^{30}$, Marie Standl ${ }^{16}$, Nikos Stratakis ${ }^{2,31}$ \\ Jordi Sunyer $^{23}$, Carel Thijs $^{29}$, Maties Torrent ${ }^{32}$, Tanja G.M. Vrijkotte ${ }^{15}$, \\ Alet H. Wijga ${ }^{33}$, Kiros Berhane ${ }^{1}$, Frank Gilliland ${ }^{1}$ and Leda Chatzi ${ }^{1,2,31}$
}

@ERSpublications

Children who have asthma or wheezing in early childhood may be at a higher risk of developing obesity http://ow.ly/HfKw30lhF3X

Cite this article as: Contreras ZA, Chen Z, Roumeliotaki T, et al. Does early onset asthma increase childhood obesity risk? A pooled analysis of 16 European cohorts. Eur Respir J 2018; 52: 1800504 [https:// doi.org/10.1183/13993003.00504-2018].

ABSTRACT The parallel epidemics of childhood asthma and obesity over the past few decades have spurred research into obesity as a risk factor for asthma. However, little is known regarding the role of asthma in obesity incidence. We examined whether early-onset asthma and related phenotypes are associated with the risk of developing obesity in childhood.

This study includes 21130 children born from 1990 to 2008 in Denmark, France, Germany, Greece, Italy, The Netherlands, Spain, Sweden and the UK. We followed non-obese children at 3-4 years of age for incident obesity up to 8 years of age. Physician-diagnosed asthma, wheezing and allergic rhinitis were assessed up to 3-4 years of age.

Children with physician-diagnosed asthma had a higher risk for incident obesity than those without asthma (adjusted hazard ratio (aHR) 1.66, 95\% CI 1.18-2.33). Children with active asthma (wheeze in the last 12 months and physician-diagnosed asthma) exhibited a higher risk for obesity (aHR 1.98, 95\% CI 1.31-3.00) than those without wheeze and asthma. Persistent wheezing was associated with increased risk for incident obesity compared to never wheezers (aHR 1.51, 95\% CI 1.08-2.09).

Early-onset asthma and wheezing may contribute to an increased risk of developing obesity in later childhood. 
Affiliations: ${ }^{1}$ Dept of Preventive Medicine, University of Southern California, Los Angeles, CA, USA. ${ }^{2}$ Dept of Social Medicine, University of Crete, Heraklion, Greece. ${ }^{3}$ Dept of Epidemiology of Allergic and Respiratory Diseases, IPLESP, INSERM, UPMC, Medical School Saint-Antoine, Paris, France. ${ }^{4}$ Dept of Pediatrics, MarienHospital Wesel, Wesel, Germany. ${ }^{5}$ Institute of Environmental Medicine, Karolinska Institute, Stockholm, Sweden. ${ }^{6}$ Center for Occupational and Environmental Medicine, Stockholm County Council, Stockholm, Sweden. ${ }^{7}$ MRC Lifecourse Epidemiology Unit, University of Southampton, Southampton General Hospital, Southampton, UK. ${ }^{8}$ Dept of Pediatrics, Division of Respiratory Medicine and Allergology, Erasmus MC, University Medical Centre Rotterdam, Rotterdam, The Netherlands. ${ }^{9}$ Dept of Epidemiology, Erasmus MC, University Medical Centre Rotterdam, Rotterdam, The Netherlands. ${ }^{10}$ Dept of Pediatrics, Division of Neonatology, Erasmus MC, University Medical Centre Rotterdam, Rotterdam, The Netherlands. ${ }^{11}$ Dept of Dermatology and Allergy Center, Odense Research Centre for Anaphylaxis (ORCA), Odense, Denmark. ${ }^{12}$ Dept of Biomedical and Neuromotor Sciences, University of Bologna, Bologna, Italy. ${ }^{13}$ Dept of Epidemiology, Lazio Regional Health Service, Rome, Italy. ${ }^{14}$ Biomax Informatics AG, Planegg, Germany. ${ }^{15}$ Dept of Public Health, Amsterdam Public Health Research Institute, Academic Medical Center, University of Amsterdam, Amsterdam, The Netherlands. ${ }^{16}$ Institute of Epidemiology I, Helmholtz Zentrum München - German Research Center for Environmental Health, Neuherberg, Germany. ${ }^{17}$ Institute and Outpatient Clinic for Occupational, Social and Environmental Medicine, Inner City Clinic, University Hospital of Munich (LMU), Munich, Germany. ${ }^{18}$ Dept of Statistics and Operational Research, University of Valencia, Valencia, Spain. ${ }^{19}$ Epidemiology and Environmental Health Joint Research Unit, FISABIO-Universitat Jaume I-Universitat de València, Valencia, Spain. ${ }^{20}$ CIBER Epidemiología y Salud Pública (CIBERESP), Madrid, Spain. ${ }^{21}$ NIHR Southampton Biomedical Research Centre, University of Southampton and University Hospital Southampton, Southampton, UK. ${ }^{22}$ Institute of Social Medicine, Epidemiology and Health Economics, Charité - Universitätsmedizin Berlin, Berlin, Germany. ${ }^{23}$ ISGlobal, Centre for Research in Environmental Epidemiology (CREAL), Barcelona, Spain. ${ }^{24}$ Universitat Pompeu Fabra (UPF), Barcelona, Spain. ${ }^{25}$ IMIM (Hospital del Mar Medical Research Institute), Barcelona, Spain. ${ }^{26}$ Dept of Paediatric Pneumology and Immunology, Charité - Universitätsmedizin Berlin, Berlin, Germany. ${ }^{27}$ Dept of Environmental Immunology/Core Facility Studies, Helmholtz Centre for Environmental Research-UFZ, Leipzig, Germany. ${ }^{28}$ The Generation R Study Group, Erasmus MC, University Medical Centre Rotterdam, Rotterdam, The Netherlands. ${ }^{29}$ Dept of Epidemiology, CAPHRI Care and Public Health Research Institute, Maastricht University Medical Centre+, Maastricht, The Netherlands. ${ }^{30}$ Julius Center for Health Sciences and Primary Care, University Medical Center Utrecht, Utrecht, The Netherlands. ${ }^{31}$ NUTRIM School of Nutrition and Translational Research in Metabolism, Maastricht University, Maastricht, The Netherlands. ${ }^{32} \mathrm{Ib}$-salut, Area de Salut de Menorca, Menorca, Spain. ${ }^{33}$ Centre for Nutrition, Prevention and Health Services, National Institute of Public Health and the Environment, Bilthoven, The Netherlands.

Correspondence: Leda Chatzi, Dept of Preventive Medicine, University of Southern California, 2001 North Soto St. 230-07, Los Angeles, CA 90032, USA. E-mail: chatzidusc.edu

\section{Introduction}

Asthma and obesity are among the most important chronic childhood disorders, both having had a parallel increase in prevalence worldwide in recent decades [1]. The concomitant rise in these conditions has stimulated research into their potential relationship yet the temporality between asthma and obesity development across the life course has not been well-established. Both disorders are thought to have their origins in early life, further complicating the assessment of a causal relationship between these conditions [2]. Although there is convincing evidence that childhood obesity increases the risk of asthma or asthma-like symptoms [3-6], there is limited evidence on whether early-life asthma could trigger obesity onset.

Two US longitudinal studies examined the potential impact of asthma on obesity development in school-aged children. The first studied 2171 non-obese kindergarten and first-grade children with 10 years follow up and showed that children with a diagnosis of asthma were at $51 \%$ increased risk of developing obesity compared to those without asthma [7]. The other examined the bidirectional association between asthma and obesity in a sample of 6452 children from kindergarten to middle school and observed that asthma was associated with increased risk for subsequent obesity onset; however, obesity or being overweight were not associated with subsequent asthma onset [8]. A pooled study of eight European birth cohorts found that children with rapid body mass index (BMI) growth in the first 2 years had a higher risk of incident asthma up to age 6 years [6], but no European studies have examined the impact of early-life asthma on incident obesity.

If most cases of childhood obesity have their origins in the preschool years, it is plausible to surmise that the asthma-obesity association may also be established in this critical time window; however, no studies to date have evaluated the impact of early-onset asthma on obesity risk in childhood. Importantly, results from previous studies need to be replicated across different, population-based longitudinal studies, examining not only asthma but associated phenotypes. In this study, we sought to leverage the rich data on early-life asthma and asthma-related comorbidities from 16 European birth cohorts to conduct a pooled analysis on the potential association between early-onset asthma and related phenotypes, and subsequent obesity risk. 


\section{Methods}

Study population

Sixteen European birth cohorts from the Mechanisms of the Development of Allergy (MeDALL) consortium and the Child Cohort Research Strategy for Europe (CHICOS) FP7 Collaborative Research Grants contributed data on asthma and related phenotypes at 3-4 years of age or younger and anthropometric information at 3-4 years of age and at any point up to 8 years of age. The participating cohorts included: ABCD [9], BAMSE [10], DARC [11], EDEN [12], Generation R [13], GINIplus [14], INMA Menorca and Valencia [15], KOALA [16], LISAplus [14], MAS [17], PIAMA [18], RHEA [19], ROBBIC Bologna and Roma [20], and SWS [21]. The recruitment period of these cohorts' span births from 1990 to 2008. Given the small sample sizes and the similarities in data collection of the ROBBIC Bologna and ROBBIC Roma cohorts, we pooled the data for these two cohorts together. All cohorts obtained informed consent from parents or legal guardians and ethical approval from the local authorised institutional review boards. A data transfer agreement document was signed by each study and anonymized data sets were transferred to the University of Crete for analysis. There were 27117 children who had available information on our exposures or outcomes. We excluded 5420 children because they did not have information for at least one asthma or asthma-related phenotype and BMI information for at least 2 follow-ups. An additional 567 children were excluded because they were obese at baseline, leaving us with 21130 children for our analyses. Detailed information on the participating cohorts is shown in table 1.

TABLE 1 Description of participating cohorts.

Study and enrolment period Total subjects $\mathrm{n} \quad$ Measurement age ${ }^{\#}$ years

Age years



Data are presented as mean $\pm S D$, unless otherwise stated. Only children with body mass index data for at least two follow-ups, who were non-obese at baseline and had information on at least one asthma or asthma-related phenotype were included. NA: not applicable. ${ }^{\#}$ : age when anthropometric measurements collected. 
We also provide information comparing the maternal and child characteristics of the children excluded from our analyses due to missing data to those included in our study (supplementary table E1).

\section{Asthma and related phenotypes}

Information on asthma, wheeze and allergic rhinitis in the participating cohorts was obtained from questionnaires that were adapted from the International Study on Asthma and Allergy in Childhood (ISAAC) and administered to parents [22]. Cohort-specific information on data collection for asthma and related phenotypes is shown in supplementary table E2. The presence of asthma at baseline (yes/no) was determined by reporting of a physician diagnosis of asthma at any point up to 3-4 years of age. Wheeze (yes/no) at baseline was based on reporting of wheezing or whistling in the chest in the past 12 months at 3-4 years of age. Early wheeze (yes/no) was based on reporting of wheezing or whistling in the chest in the past 12 months assessed at any time in infancy ( $0-2$ years of age). Allergic rhinitis (yes/no) at baseline was based on reporting of rhinitis (sneezing or a runny or blocked nose when not a cold or flu) in the past 12 months at 3-4 years of age. Asthma history was based on responses to the baseline asthma and wheeze questions and categorised as: 1) have baseline asthma and baseline wheeze (active asthma); 2) have baseline asthma, but no baseline wheeze; 3) have no baseline asthma, but have baseline wheeze; and 4) have no baseline asthma and no baseline wheeze (reference category). Based on previous studies, we defined distinct wheezing phenotypes throughout childhood [23, 24]. Wheezing history was constructed using responses to the baseline wheeze and early wheeze questions, and categorised as: 1) have early wheeze and baseline wheeze (persistent wheezing); 2) have no early wheeze, but have baseline wheeze (late-onset wheezing); 3) have early wheeze, but no baseline wheeze (transient wheezing); and 4) have no early wheeze and no baseline wheeze (never wheezing; the reference category). Since we lacked information on IgE sensitisation, we created a measure of asthma and allergic rhinitis comorbidity based on responses to the baseline asthma and allergic rhinitis questions, and categorised as: 1) have baseline asthma and allergic rhinitis; 2) have baseline asthma and no allergic rhinitis; and 3) have no baseline asthma and no allergic rhinitis. We also created a combined measure of the joint impact of asthma and medication use, categorised as: 1) yes asthma/yes medication use; 2) yes asthma/no medication use; 3) no asthma/yes medication use; and 4) no asthma/no medication use (reference category).

\section{Obesity}

Cohorts provided weight and height information based on clinical examinations, health records, or parental-reported questionnaires at 3-4 years of age and at least one other time point between 5-8 years of age. BMI was calculated as weight $(\mathrm{kg})$ divided by height $(\mathrm{m})$ squared. Since our primary outcome was incident obesity we restricted the analysis to normal weight or overweight children at baseline. Obese status was defined according to the 2012 Cole-International Obesity Task Force age and sex-specific cut-offs [25]. We also used the 2007 World Health Organization cut-offs for obesity in sensitivity analyses [26, 27].

\section{Covariate assessments}

Potential covariates were defined as similarly as possible among the cohorts. Information on maternal smoking during pregnancy (yes/no), birthweight (g) and child sex (male or female) was collected through interviews or self-administered questionnaires, ad hoc measurements, birth records, or medical registries. Information on maternal education (cohort-specific definitions of low, medium or high), any breastfeeding (months), parity (primiparous or multiparous), any dampness or mold in the home at $0-4$ years (yes/no), passive smoke (childhood exposure to smoking by others in the household at 0-4 years, yes/no), medication for asthma or breathing problems in the last 12 months at 3-4 years of age (yes/no), parental history of asthma and pets in the home during infancy (0-2 years, yes/no) was obtained via interviews or self-administered questionnaires. Birthweight was subsequently categorised as low $(<2500 \mathrm{~g})$, normal (2500-3999 g), or high $(4000+\mathrm{g})$ and breastfeeding was categorised as $<3$ months and $\geqslant 3$ months [28, 29]. Physical activity was available for only three out of 16 cohorts and thus we were not able to include it as a potential covariate. More detailed information on the type of medication use was available for four cohorts (BAMSE, DARC, PIAMA and ROBBIC) and defined as use of inhaled corticosteroids (ICS) ever at 3-4 years of age (yes/no).

\section{Statistical analyses}

Our main analysis was a pooled analysis in which we combined the data from 16 different cohorts. To account for potential differences between cohorts we introduced a "random effect for cohort" or a "fixed effect for cohort" (i.e. an indicator variable). Since we observed no differences between estimates derived from random effects and fixed effects models, we present results from fixed effects models as they are more likely to provide unbiased estimates in large samples [30]. We estimated adjusted hazard ratios (aHRs) and 95\% CIs for the associations of childhood asthma and related phenotypes at baseline with 
obesity incidence during follow-up using Cox proportional hazards models with a sex-specific baseline hazard. We used age at study visit as the time scale in our analysis and onset of obesity was defined as happening when the child first become obese during follow-up (at the midpoint of the follow-up period between the visit when they were not obese and the subsequent visit when they were assessed as obese). Children who did not become obese during follow-up were censored at the end of study follow-up or when lost to follow-up. Selection of confounders for adjustment was based on directed acyclic graphs (DAGs), informed by previous knowledge and constructed using DAGitty version 3.0 (supplementary figure E1) [31]. The confounders included in final models were age at baseline, smoking during pregnancy, passive smoke, parity, maternal education, parental asthma, breastfeeding and birthweight. All cohorts had information available for these confounders, with the exception of maternal education and smoking during pregnancy for the MAS cohort and parity information for the INMA Menorca cohort. Children with missing covariate information were included in the analysis using the missing indicator method, in which missing values were used as an additional group in categorical variables. Though dampness or mold in the home and pets in the home during infancy were potential confounders based on our DAG, further adjustment for these covariates did not substantially change effect estimates and these variables were thus not included in final models. Since medication use may be a potential mediator of the asthma and obesity relationship we explored its role further by conducting several analyses, as follows: 1) we examined the impact of medication use on obesity incidence without adjusting for asthma status; 2) we assessed the impact of asthma and medication use on incident obesity when mutually adjusting for both in the same model; and 3) we modelled the joint impact of asthma and medication use on obesity incidence using a combined measure of asthma and medication use. We confirmed that the proportional hazards assumption was met using Schoenfeld residuals.

We performed several sensitivity analyses. First, we adjusted for overweight at baseline to determine whether our results were disproportionately impacted by children who were in this category. Secondly, we restricted our sample to those who were normal weight at baseline and conducted separate analyses examining the incidence of overweight or obesity, as well as obesity only, to further assess the impact of the overweight at baseline. Thirdly, we adjusted for baseline BMI as a continuous variable to assess whether initial BMI status impacts our results. Fourthly, we excluded children who developed asthma during follow-up to assess whether our results were impacted by new asthma cases. Fifthly, we assessed whether effects vary according to factors (sex, birthweight, parental asthma, maternal education, asthma medication and breastfeeding (never, $<6$ months, 6+ months)) that may make certain groups more vulnerable by conducting stratified analyses and introducing interaction terms [32]. Sixthly, we repeated our analysis using the World Health Organization (WHO) cut-offs for obesity to compare our results using an alternative definition of obesity. Seventhly, we assessed whether adjustment for height at the end of follow-up changed our results to tease apart whether changes in weight are mainly driving the association. Eighthly, we restricted our definition of obesity to children who were obese for at least two visits. Lastly, we explored whether estimates differed in complete-case analyses.

Furthermore, we performed an individual participant meta-analysis as a supplementary analysis to assess whether our results were consistent with those from our pooled analyses. We used Cox proportional hazards models with a sex-specific baseline hazard and the aforementioned set of confounders to estimate cohort-specific aHRs and 95\% CIs for the associations of baseline asthma and other related phenotypes with obesity incidence during follow-up. We combined cohort-specific estimates using random effects meta-analysis in which the weight assigned to each study was based on both the within-study and between-study variability. We examined heterogeneity between cohort-specific estimates with the $\mathrm{I}^{2}$ statistic and the $X^{2}$ test from Cochran's Q. We also tested the robustness of the results by repeating the meta-analyses and excluding one cohort at a time. Finally, we explored potential heterogeneity by region of participating cohorts, based on the United Nations' classification (Southern Europe, Western Europe and Northern Europe), by conducting meta-regression analyses. All analyses were performed using Stata 14.2 software (StataCorp LLC, College Station, TX, USA).

\section{Results}

The analysis included 21130 children at cohort entry with a mean age at baseline of $4.1 \pm 0.6$ years (table 1). The maternal and child characteristics of our total study population, as compared to those that were excluded from our study due to missing data, are displayed in supplementary table E1. Overall, we did not observe any substantial differences in characteristics between these groups except for a slightly higher prevalence of low maternal education $(60.2 \%$ versus $55.0 \%)$ and shorter breastfeeding duration (63.1\% versus $67.7 \%$ ) in excluded children. Overall, the prevalence of physician-diagnosed asthma at baseline was $6.0 \%$, while the prevalence of baseline wheeze and early wheeze was $12.5 \%$ and $18.1 \%$, respectively (table 2 ). About $13.7 \%$ of children had allergic rhinitis. The prevalence of asthma and related phenotypes for each cohort at baseline is shown in supplementary table E3. The number of follow-ups and age at end of 
TABLE 2 Association of early-onset asthma and asthma-related phenotypes with incident obesity up to age 8 years in a pooled sample of 21130 children from 16 European birth cohorts

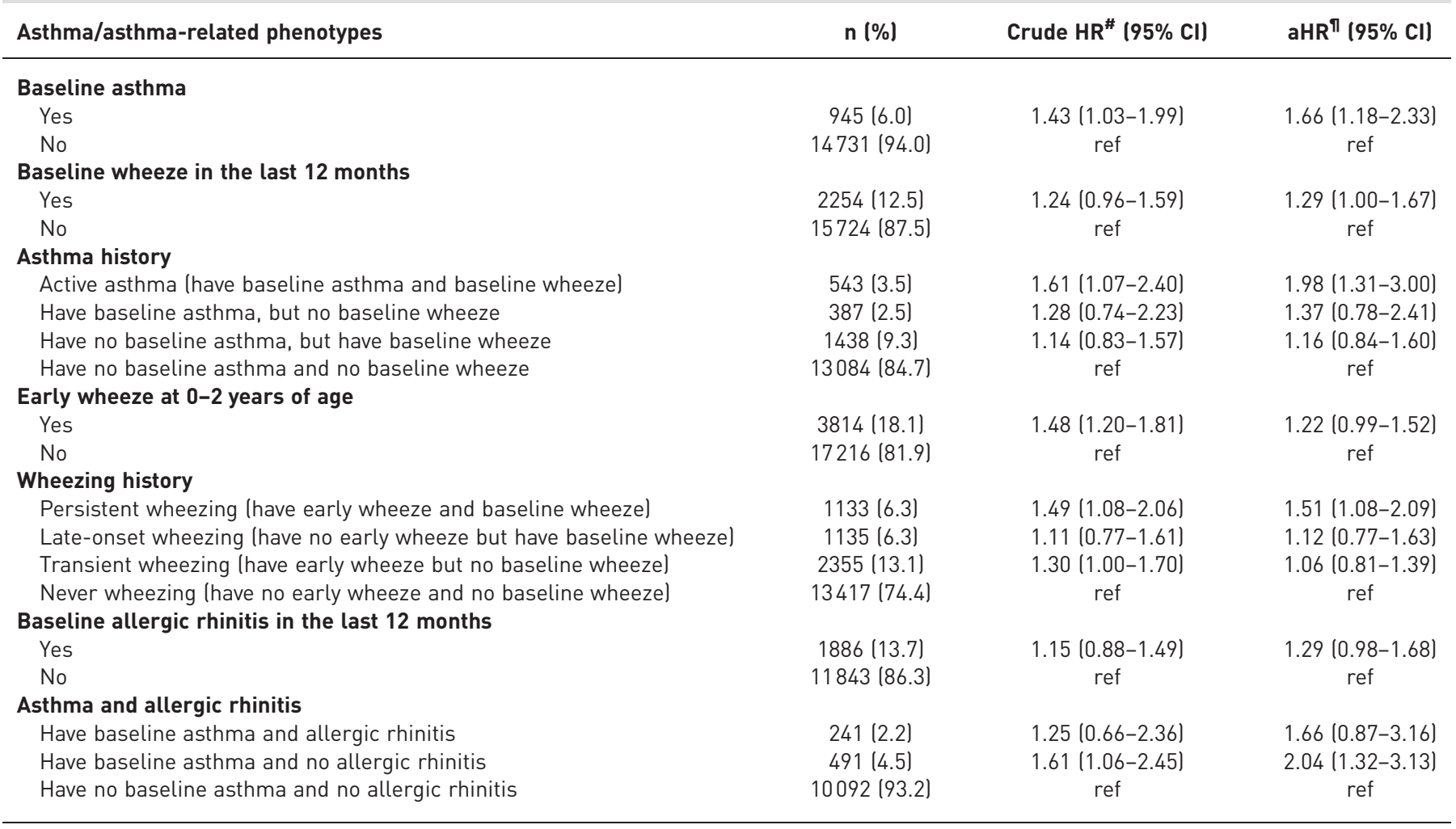

aHR: adjusted hazard ratio; ref: reference category. ${ }^{\#}$ : the Cox proportional hazards model used a sex-specific baseline hazard; ${ }^{\text {ๆ: }}$ the Cox proportional hazards model was adjusted for cohort, age at baseline, smoking during pregnancy, passive smoke, parity, maternal education, parental asthma, breastfeeding and birthweight with a sex-specific baseline hazard.

follow-up varied by cohort with an overall mean age at endpoint of $7.1 \pm 1.2$ years and 3.2 visits on average (table 1). During follow-up, 483 children (2.3\%) developed obesity. Cohort-specific prevalences of baseline obesity and incident obesity are shown in supplementary table E4, while baseline maternal and child characteristics by cohort are presented in supplementary table E5.

The pooled analysis results for the association between asthma and related phenotypes at baseline and incident obesity at follow-up are displayed in table 2. Children with physician-diagnosed asthma had a $66 \%$ higher risk of incident obesity than those without an asthma diagnosis (aHR 1.66, 95\% CI 1.18-2.33; table 2). Children with wheeze at baseline had an increased risk of obesity compared to those with no baseline wheeze (aHR 1.29, 95\% CI 1.00-1.67). Children with active asthma exhibited an even greater risk for developing obesity (aHR 1.98, 95\% CI 1.31-3.00) than those without asthma and wheeze. Wheeze in infancy had a similar impact on incident obesity as baseline wheeze (aHR 1.22, 95\% CI 0.99-1.52). However, for wheezing history, compared to children who never wheezed, obesity risk was more pronounced for children with persistent wheezing (aHR 1.51, 95\% CI 1.08-2.09) than late-onset wheezing (aHR 1.12, 95\% CI 0.77-1.63) or transient wheezing (aHR 1.06, 95\% CI 0.81-1.39). Allergic rhinitis was positively associated with obesity onset (aHR 1.29, 95\% CI 0.98-1.68). Children with asthma and no allergic rhinitis had a higher risk of incident obesity than those without asthma (aHR 2.04, 95\% CI $1.32-3.13)$.

The results for the joint associations of asthma and medication use with incident obesity are shown in table 3. Medication use for asthma or breathing difficulties increased the risk of incident obesity (aHR 1.37, 95\% CI 1.04-1.80). When adjusting for asthma status, the impact of medication use on obesity was attenuated slightly and was no longer statistically significant (aHR 1.23, 95\% CI 0.91-1.66). In contrast, adjustment for medication use did not change the significant positive association between asthma and incident obesity (aHR 1.57, 95\% CI 1.04-2.37). When assessing the combined impact of asthma and medication use, we found that compared to children with no asthma and no medication use, asthmatic children taking medication had a higher risk of incident obesity (aHR 1.91, 95\% CI 1.25-2.92) than 
TABLE 3 Association of early-onset asthma and asthma medication use with incident obesity up to age 8 years in a pooled sample of 11788 children from eight European birth cohorts

\begin{tabular}{|c|c|c|}
\hline Asthma/asthma-related phenotypes & n $(\%)$ & $\operatorname{aHR}^{\#}(95 \% \mathrm{CI})$ \\
\hline \multicolumn{3}{|c|}{$\begin{array}{l}\text { Baseline medication use for asthma or breathing difficulties } \\
\text { in the last } 12 \text { months }\end{array}$} \\
\hline Yes & 2062 (17.5) & $1.37(1.04-1.80)$ \\
\hline No & 9726 (82.5) & ref \\
\hline \multicolumn{3}{|c|}{$\begin{array}{l}\text { Baseline medication use for asthma or breathing difficulties } \\
\text { in the last } 12 \text { months adjusted for baseline asthma }\end{array}$} \\
\hline Yes & $1918(16.9)$ & $1.23(0.91-1.66)$ \\
\hline No & $9416(83.1)$ & ref \\
\hline \multicolumn{3}{|c|}{$\begin{array}{l}\text { Baseline asthma adjusted for baseline medication use for } \\
\text { asthma or breathing difficulties in the last } 12 \text { months }\end{array}$} \\
\hline Yes & $721(6.4)$ & $1.57(1.04-2.37)$ \\
\hline No & $10614(93.6)$ & ref \\
\hline \multicolumn{3}{|l|}{ Baseline asthma and medication use } \\
\hline Yes asthma/yes medication use & $505(4.5)$ & $1.91(1.25-2.92)$ \\
\hline Yes asthma/no medication use & 216 (1.9) & $1.65(0.73-3.73)$ \\
\hline No asthma/yes medication use & 1413 (12.5) & $1.24(0.90-1.69)$ \\
\hline No asthma/no medication use & 9201 (81.2) & ref \\
\hline
\end{tabular}

aHR: adjusted hazard ratio; ref: reference category. ${ }^{\#}$ : the Cox proportional hazards model was adjusted for cohort, age at baseline, smoking during pregnancy, passive smoke, parity, maternal education, parental asthma, breastfeeding and birthweight with a sex-specific baseline hazard.

asthmatic children not taking medication (aHR 1.65, 95\% CI 0.73-3.73) and nonasthmatic children taking medication (aHR 1.24, 95\% CI 0.90-1.69). ICS use was positively associated with incident obesity both with adjustment for asthma (aHR 1.42, 95\% CI 0.82-2.26) and without (aHR 1.45, 95\% CI 0.97-2.18).

The cohort-specific estimates and combined estimates from the random effects meta-analysis for the association between asthma and related phenotypes and incident obesity are presented in figure 1 . Cohort-specific estimates were not estimable for INMA Menorca and LISA for physician-diagnosed asthma, and for DARC for allergic rhinitis, due to zero exposed incident obesity cases within the respective cohorts. The combined effect estimates were consistent with those of our pooled analyses, albeit stronger in magnitude. There was no evidence of significant heterogeneity between cohorts $\left(\mathrm{I}^{2}=0, \mathrm{p}\right.$-value $>0.48$ ) or by geographical region of cohorts. The results remained similar when omitting one cohort at a time (supplementary table E6).

When adjusting for overweight at baseline in our pooled analysis, the associations we observed were attenuated slightly but remained significant (supplementary table E7). After restricting our sample to normal weight at baseline, observed associations were attenuated and no longer statistically significant, but were still indicative of a positive association for incident obesity (supplementary table E8). After adjustment for baseline BMI, our observed associations were similarly attenuated and nonsignificant, albeit still positively associated with incident obesity (supplementary table E9). When excluding new asthma cases during follow-up ( $\mathrm{n}=448)$, the association between asthma and incident obesity did not change (aHR 1.65, 95\% CI 1.17-2.32). There was no evidence of interaction or differences in stratified analyses between asthma and sex, birthweight, parental asthma, maternal education, asthma medication and breastfeeding. Adjustment for child's height at the end of follow-up did not change the relationship between asthma and incident obesity (aHR 1.64, 95\% CI 1.17-2.31). We observed similar results when using the WHO definition of obesity and slightly attenuated results when we defined obesity as remaining obese for at least two visits (data not shown). No differences in our results were observed in complete-case analyses (data not shown).

\section{Discussion}

We found that early-onset asthma and wheezing were associated with a higher incidence of childhood obesity. The evidence for allergic rhinitis was less strong, but still indicative of a higher risk for obesity. This is the only longitudinal, multicenter study to date that has examined the impact of early-onset asthma on obesity development in children. The inclusion of birth cohorts from numerous European countries and the absence of heterogeneity between our cohort-specific effect estimates supports the robustness and generalisability of our results. 
a)



Study Participants HR $(95 \% \mathrm{CI}) \quad \%$ weight

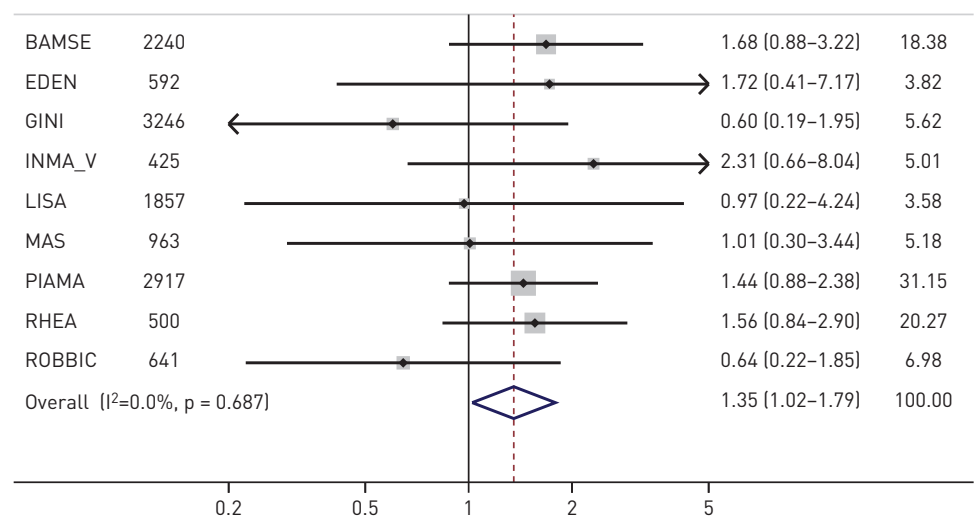

FIGURE 1 Results of individual participant meta-analyses for the association of early-onset asthma and asthma-related phenotypes with incident obesity up to age 8 years. a) Association between baseline asthma and incident obesity. b) Association between baseline wheeze in the last 12 months at 3-4 years of age and incident obesity. c) Association between baseline allergic rhinitis in the last 12 months and incident obesity. Hazard ratios ( $\mathrm{HR} ; 95 \% \mathrm{Cl}$ ) by cohort were obtained by using Cox proportional hazards models adjusted for age at baseline, smoking during pregnancy, passive smoke, parity, maternal education, parental asthma, breastfeeding and birthweight with a sex-specific baseline hazard. Combined estimates were obtained by using a random-effects meta-analysis. The squares represent the point estimate of each study and the size of the square is proportional to the weight assigned to each cohort based on both the within-study and between-study variability. Horizontal lines denote $95 \% \mathrm{Cls}$ and diamonds represent overall estimates. The arrows on some of the Cls denote that the upper or lower bound of the confidence interval is past the range of the values shown on the $\mathrm{x}$-axis. 
Although many studies have assessed the effect of childhood obesity on asthma development, the temporal order between these conditions remains unclear, largely due to the scarcity of studies examining whether asthma can affect obesity onset. We were able to assess the impact of early-life diagnosis of asthma on subsequent childhood obesity risk. Our study corroborates the findings of two previous US longitudinal studies that observed an increased risk of obesity in school-aged children with asthma [7, 8]. The diagnosis of asthma in young children is difficult and physicians may be hesitant to make the diagnosis at an early age. As such, wheezing in childhood is often used as an indicator of future asthma development. Although this measure lacks specificity, our finding that the risk of obesity is highest in children with persistent wheeze is important because children who have persistent wheeze are more likely to develop early-onset asthma than those with transient or late-onset wheeze [24,33]. Additionally, our results for active asthma suggest that asthmatic children who are currently experiencing wheezing symptoms are at highest risk. The only previous study to have assessed the impact of active asthma on obesity development found similar results [7].

Recent studies suggest that there are at least two distinct asthma phenotypes associated with obesity, early-onset asthma complicated by obesity and late-onset asthma arising because of obesity. Early-onset asthma is characterised by a higher prevalence of allergic disease and T-helper cell Type-2 (Th2) inflammation, whereas late-onset asthma has a lower prevalence of allergic disease and is less related to Th2 inflammation [34]. It is possible that, in children with the severe allergic asthma phenotype, asthma itself may lead to obesity although this hypothesis has not been well-explored in studies to date [2, 35]. However, in our study we found a stronger effect estimate for asthmatics without allergic rhinitis than those with rhinitis. Although the presence of allergic rhinitis is strongly associated with allergic asthma, these results are inconclusive as they are not based on atopy measures (i.e. specific IgE measures). Further studies are needed in this direction to differentiate childhood obesity risk between IgE-sensitised and IgE-unsensitised individuals, and those with allergic asthma and nonallergic asthma.

The association between asthma and obesity may also be explained by common biological pathways that promote the development of these conditions in early life. A recent study in mice found that the chitinase 3-like protein 1 (Chi311) plays a key role in white adipose tissue accumulation and lung Th2 inflammation. A high fat diet and aeroallergen challenge increased the expression of white adipose tissue and pulmonary Chi311, suggesting that a high-fat diet contributes to visceral adiposity and asthma by stimulating the Chi3l1 pathway, and that asthma itself can increase obesity [36]. Other hypothesised pathways underlying asthma and obesity include systemic inflammation, adipokine dysregulation and shared genetics or epigenetic changes, as well as the gut microbiome; however, the evidence for these pathways is limited [34, 37].

Common lifestyle factors related to both asthma and obesity may partially explain their association. For instance, studies have shown that asthmatic children have lower physical activity levels and poorer sleep, which are also risk factors for obesity [38]. We lacked information on a child's physical activity and sleep and thus we could not test this hypothesis in our study. Additionally, given the early-life origin of these disorders, shared in utero exposures may also account for the relationship we observe. For instance, prenatal diet, maternal obesity and early infant growth have been associated with increased risk of both disorders [37, 39].

Weight gain due to medication use for asthma has also been posited as a potential mechanism linking asthma and obesity. Two prospective studies found greater increases in BMI in asthmatic children on higher doses of ICS compared to those on lower doses [40,41]. Medication use itself may increase obesity risk or it may be that children with more severe asthma, who are also on higher doses of steroids, have reduced activity levels or other factors that predispose them to obesity. In our study, we lacked detailed information on medication dosage and asthma severity. However, we observed the strongest risk of obesity for asthma with medication use than asthma without medication use or medication use in the absence of asthma. When we mutually adjusted for asthma and medication, the positive association between medication use and incident obesity was attenuated, but the association between asthma and obesity did not change markedly. For the four cohorts with available data, inhaled steroid use was positively associated with obesity even after adjustment for asthma. Thus, our results suggest that the joint presence of asthma and medication use has the greatest impact on obesity risk and that the association between asthma and obesity cannot be fully explained by medication use. In addition, our results for the impact of medication use on obesity, though inconclusive, suggest that it can increase the risk of obesity independently of asthma status.

Strengths of our study include the population-based longitudinal design and the inclusion of individual participant data from several European birth cohorts with different background characteristics and behaviours. Other strengths include the large sample size of the study and the standardised exposure definitions and harmonised covariate information. Given the discrepancy in the literature on the definition 
of childhood obesity [42], we tested three definitions and found comparable results that suggested our findings are not subject to different definition criteria and represent a true onset of obesity.

While novel and large in scale, our study has several limitations. Some cohorts did not have data available for all confounding variables and the use of an extra category for missing confounder information could have introduced residual confounding. However, when we performed complete case analyses, we observed comparable results. Although we controlled for breastfeeding, there is the possibility of residual confounding by the mother or child's early-life dietary patterns and energy intake [37]. We may also have uncontrolled confounding due to maternal BMI, since studies suggest that pre-pregnancy obesity may increase the risk of childhood asthma; however, our study lacks data for adjustment for maternal obesity status [43]. When we excluded overweight children from the baseline group, or when we adjusted for baseline BMI, the association between asthma and obesity was attenuated but results were in the same direction, possibly due to the few years of follow-up and low obesity incidence in our study. Therefore, based on our results, we cannot exclude the possibility of reverse causality; however, in analyses in which we adjusted for overweight at baseline, we still observed significantly elevated effect estimates. We acknowledge that there are substantial variations in the prevalence of asthma and wheezing by country because asthma may be more readily diagnosed in some countries and underdiagnosed in others [44]. In addition, the prevalence of obesity varies by country, which is partly explained by differences in diet, physical activity and sedentary behaviours [45, 46]. However, the absence of heterogeneity in the study-specific estimates suggests that any potential misclassification of exposure is not a major source of bias. The children excluded from our study due to missing data were more likely to have lower socioeconomic status (SES) and shorter breastfeeding duration, which are both thought to be risk factors for asthma and obesity. Since both factors are positively associated with our exposure and outcome, selection bias resulting from their exclusion would likely negatively bias our results. Given the magnitude of the association between asthma and incident obesity we observed, it is unlikely that this potential source of bias is a major threat to the validity of our study. Asthma and related comorbidities were assessed by parental report, which could introduce misclassification, but questionnaires were well-validated based on the ISAAC study [22]. We also had less power in our analyses in which we combined the presence of multiple asthma and asthma-related conditions since not all children had complete data on these conditions. Since anthropometric measures were based on a mix of parental report and clinical assessment information, there is the potential for misclassification of obesity in our study. However, we do not expect any misclassification of reported weight information to be informed by asthma status and thus the resulting bias would be towards the null.

This large, multi-center longitudinal study suggests that early-onset asthma and wheezing may contribute to an increased incidence of developing obesity in later childhood. Our findings lend support to the need for further investigation of the factors driving the increased risk of obesity in asthmatic children to better tailor future obesity prevention efforts.

Acknowledgements: BAMSE: We thank all the children and their parents for participating in the BAMSE cohort and the nurses and other staff members working in the BAMSE project. EDEN: We acknowledge the commitment of the EDEN mother-child cohort study group: I. Annesi-Maesano, J.Y. Bernard, J. Botton, M.A. Charles, P. Dargent-Molina, B. de Lauzon-Guillain, P. Ducimetière, M. de Agostini, B. Foliguet, A. Forhan, X. Fritel, A. Germa, V. Goua, R. Hankard, B. Heude, M. Kaminski, B. Larroque ${ }^{\dagger}$, N. Lelong, J. Lepeule, G. Magnin, L. Marchand, C. Nabet, F. Pierre, R. Slama, M.J. Saurel-Cubizolles, M. Schweitzer, O. Thiebaugeorges. ROBBIC: We thank all the field workers and the families for their contribution to the study. SWS: we thank the members of the Southampton Women's Survey study group and the many participants in the SWS for their contribution to the study.

Author contributions: Z.A. Contreras, T. Roumeliotaki and L. Chatzi had full access to all the data in the study and take responsibility for the integrity of the data and the accuracy of the data analysis. Study concept and design: L. Chatzi; acquisition of data: all authors; statistical analysis: Z.A. Contreras and T. Roumeliotaki; interpretation of data: all authors; drafting of the manuscript: Z.A. Contreras and L. Chatzi; critical revision of the manuscript for important intellectual content: all authors; study supervision: L. Chatzi.

Conflict of interest: L. Duijts reports grants from European Union's Horizon 2020 co-funded programme ERA-Net on Biomarkers for Nutrition and Health (ERA HDHL) (the ALPHABET project (number 696295; 2017) and ZonMW The Netherlands (number 529051014; 2017)) and grants from the European Union's Horizon 2020 programme (the LIFECYCLE project, grant agreement number 733206; 2016), during the conduct of the study. B. Gerhard reports grants from the EU FP7, during the conduct of the study. H. Inskip reports grants (salary) from the UK Medical Research Council, grants from the British Heart Foundation, the UK Food Standards Agency, the British Lung Foundation, Arthritis Research UK, the NIHR Southampton Biomedical Research Centre and the European Union's Seventh Framework Programme (FP7/2007-2013, project EarlyNutrition under grant agreement number 289346), during the conduct of the study; and members of H. Inskip's team have received funding from Nestec, Abbott Nutrition and Danone Nutricia for various projects to which she is linked, but she has received no direct funding herself. S. Lau reports grants and personal fees (honoraria) from Symbiopharm and Allergopharma, personal fees (honoraria) and non-financial support (advisory board work) from DBV, personal fees from ALK, and non-financial support (advisory 
board work) from Boehringer and Merck, outside the submitted work. D. Maier reports grants from the European Commission (FP7 MeDALL, 261357), during the conduct of the study.

Support statement: This work was partially supported by the Southern California Environmental Health Sciences Center (grant \# P30ES007048) funded by the National Institute of Environmental Health Sciences (PI F. Gilliland). Z.A. Contreras was supported by a grant from the NIH (T32 ES013678). MeDALL and CHICOS Projects: the research leading to these results has received funding from the European Community's Seventh Framework Programme (Health-F2 grant agreements 261357 and 241604). ABCD cohort: data of the Amsterdam Born Children and their Development cohort study used in this research was in part supported by funds from the Netherlands Organisation for Health Research and Development (ZonMw 40-00812-98-11010). The study sponsors had no role in study design, data analysis, interpretation of data, or writing of this report. BAMSE: we acknowledge all funding sources (the Swedish Research Council, the Swedish Heart and Lung Foundation, the Swedish Research Council for Working Life and Social Welfare, the Swedish Asthma and Allergy Association Research Foundation, the Swedish Research Council Formas, Stockholm County Council and the European Commission's Seventh Framework 29 Programme MeDALL under grant agreement number 261357). EDEN: we acknowledge all the funding sources for the EDEN study (the Foundation for Medical Research (FRM), the National Agency for Research (ANR), the National Institute for Research in Public health (IRESP: TGIR cohorte santé 2008 programme), the French Ministry of Health (DGS), the French Ministry of Research, the INSERM Bone and Joint Diseases National Research (PRO-A) and Human Nutrition National Research Programmes, Paris-Sud University, Nestlé, the French National Institute for Population Health Surveillance (InVS), the French National Institute for Health Education (INPES), the European Union FP7 programme (FP7/2007-2013, HELIX, ESCAPE, ENRIECO and MeDALL projects), the Diabetes National Research Program (through a collaboration with the French Association of Diabetic Patients (AFD)), the French Agency for Environmental Health Safety (now ANSES), Mutuelle Générale de l'Education Nationale a complementary health insurance (MGEN), the French national agency for food security and the French speaking association for the study of diabetes and metabolism (ALFEDIAM)). The Generation R Study: the Generation R study is made possible by financial support from the Erasmus Medical Center Rotterdam, the Erasmus University Rotterdam, the Netherlands Organization for Health Research and Development (ZonMw), the Netherlands Organisation for Scientific Research (NWO), the Ministry of Health, Welfare and Sport and the Ministry of Youth and Families. The project received funding from the European Union's Horizon 2020 research and innovation programme (the LIFECYCLE project, grant agreement number 733206; 2016), the European Research Council (ERC-2014-CoG-648916) and from the cofunded ERA-Net on Biomarkers for Nutrition and Health (ERA HDHL), Horizon 2020 (grant agreement number 696295; 2017), ZonMW The Netherlands (number 529051014; 2017), Science Foundation Ireland (no SFI/16/ERA-HDHL/3360) and the European Union (the ALPHABET project). The researchers are independent from the funders. The study sponsors had no role in study design, data analysis, interpretation of data, or writing of this report. INMA: data used for this research was provided by the INMA-Environment and Childhood Project (www.proyectoinma.org). This study was funded by grants from the Instituto de Salud Carlos III (Red INMA G03/176 and CB06/02/0041), the Spanish Ministry of Health (FIS-PIO41436, PI06/0867, PI081151, and FIS-FEDER 03/1615, 04/1112, 04/1931, 05/1079, 05/1052, 06/1213, 07/0314 and 09/02647), the Generalitat de Catalunya-CIRIT 1999SGR00241, the Conselleria de Sanitat Generalitat Valenciana, the Department of Health of the Basque Government (2005111093 and 2009111069) and the Provincial Government of Gipuzkoa (DFG06/004 and DFG08/001). KOALA: the collection of the data from the KOALA Birth Cohort study used in this analysis was financially supported by Friesland Foods (now FrieslandCampina), the Netherlands Asthma Foundation (grant numbers 3.2.07.022 and 3.2.03.48), the Netherlands Heart Foundation (grant number 2014 T037), the Netherlands Organization for Health Research and Development (ZonMw Prevention Program number 1.210-00-090), the Triodos Foundation, the Phoenix Foundation, the Raphaël Foundation, the Iona Foundation and the Foundation for the Advancement of Heilpedagogie, all in the Netherlands. MAS: the MAS birth cohort was funded by grants from the German Federal Ministry of Education and Research (BMBF; reference numbers 07015633, 07 ALE 27, 01EE9405/5, 01EE9406) and the German Research Foundation (DFG; reference number KE 1462/2-1). PIAMA: the PIAMA study was funded by the Netherlands Organisation for Health Research and Development, the Netherlands Organisation for Scientific Research, the Netherlands Asthma fund, the Netherlands Ministry of Spatial Planning, Housing and the Environment and the Netherlands Ministry of Health, Welfare and Sport. RHEA: the Rhea project was financially supported by European projects (EU FP6-003-Food-3-NewGeneris, contract number 16320; EU FP6 STREP Hiwate, contract number 36224; EU FP7 ENV.2007.1.2.2.2, project number 211250 ESCAPE; EU FP7-2008-ENV-1.2.1.4 Envirogenomarkers, contract number 226756; EU FP7-HEALTH-2009-single stage CHICOS, contract number 241604; EU FP7 ENV.2008.1.2.1.6, proposal number 226285 ENRIECO; EU-FP7, proposal number 264357 MeDALL; EU-FP7-HEALTH-2012, proposal number 308333 HELIX) and the Greek Ministry of Health (Program of Prevention of Obesity and Neurodevelopmental Disorders in Preschool Children, Heraklion District, Crete, Greece: 2011-2014; "Rhea Plus": Prevention Program of Environmental Risk Factors for Reproductive Health, and Child Health: 2012-2015). ROBBIC: data from the Rome cohort was in part supported by funds from the Italian Ministry of Health (Programma speciale ex art. 12, comma 2, lettera b) del D. Lgs. 502/92, 2001, 2003). SWS: the Southampton Women's Survey is supported by grants from the Medical Research Council, the British Heart Foundation, the Food Standards Agency, the British Lung Foundation, Arthritis Research UK, the NIHR Southampton Biomedical Research Centre, the University of Southampton and University Hospital Southampton NHS Foundation Trust and the European Union's Seventh Framework Programme (FP7/2007-2013, project EarlyNutrition under grant agreement number 289346). Funding information for this article has been deposited with the Crossref Funder Registry.

\section{References}

Eder W, Ege MJ, von Mutius E. The asthma epidemic. N Engl J Med 2006; 355: 2226-2235.

2 Martinez FD, Guerra S. Early origins of asthma: role of microbial dysbiosis and metabolic dysfunction. Am J Respir Crit Care Med 2017; 197: 573-579.

3 Chen Y-C, Liou T-H, Chen P-C, et al. Growth trajectories and asthma/rhinitis in children: a longitudinal study in Taiwan. Eur Respir J 2017; 49: 1600741.

4 Gilliland FD, Berhane K, Islam T, et al. Obesity and the risk of newly diagnosed asthma in school-age children. Am J Epidemiol 2003; 158: 406-415. 
5 Gold DR, Damokosh AI, Dockery DW, et al. Body-mass index as a predictor of incident asthma in a prospective cohort of children. Pediatr Pulmonol 2003; 36: 514-521.

6 Rzehak P, Wijga AH, Keil T, et al. Body mass index trajectory classes and incident asthma in childhood: results from 8 European birth cohorts - a Global Allergy and Asthma European Network initiative. J Allergy Clin Immunol 2013; 131: 1528-1536.

7 Chen Z, Salam MT, Alderete TL, et al. Effects of childhood asthma on the development of obesity among school-aged children. Am J Respir Crit Care Med 2017; 195: 1181-1188.

8 Green TL. Examining the temporal relationships between childhood obesity and asthma. Econ Hum Biol 2014; 14: $92-102$.

9 van Eijsden M, Vrijkotte TG, Gemke RJ, et al. Cohort profile: the Amsterdam born children and their development (ABCD) study. Int J Epidemiol 2011; 40: 1176-1186.

10 Wickman M, Kull I, Pershagen G, et al. The BAMSE project: presentation of a prospective longitudinal birth cohort study. Pediatr Allergy Immunol 2002; 13: Suppl. 15, 11-13.

11 Johnke H, Vach W, Norberg LA, et al. A comparison between criteria for diagnosing atopic eczema in infants. Br J Dermatol 2005; 153: 352-358.

12 Heude B, Forhan A, Slama R, et al. Cohort profile: the EDEN mother-child cohort on the prenatal and early postnatal determinants of child health and development. Int J Epidemiol 2016; 45: 353-363.

13 Jaddoe VW, van Duijn CM, van der Heijden AJ, et al. The Generation R study: design and cohort update 2010. Eur J Epidemiol 2010; 25: 823-841.

14 Heinrich J, Bruske I, Schnappinger M, et al. Die zwei deutschen Geburtskohorten GINIplus und LISAplus [Two German birth cohorts: GINIplus and LISAplus]. Bundesgesundheitsbl 2012; 55: 864-874.

15 Guxens M, Ballester F, Espada M, et al. Cohort profile: the INMA-infancia y medio ambiente-(environment and childhood) project. Int J Epidemiol 2012; 41: 930-940.

16 Kummeling I, Thijs C, Penders J, et al. Etiology of atopy in infancy: the KOALA birth cohort study. Pediatr Allergy Immunol 2005; 16: 679-684.

17 Nickel R, Niggemann B, Grüber C, et al. How should a birth cohort study be organised? Experience from the German MAS cohort study. Paediatr Respir Rev 2002; 3: 169-176.

18 Wijga AH, Kerkhof M, Gehring U, et al. Cohort profile: the prevention and incidence of asthma and mite allergy (PIAMA) birth cohort. Int J Epidemiol 2014; 43: 527-535.

19 Chatzi L, Leventakou V, Vafeiadi M, et al. Cohort profile: the mother-child cohort in Crete, Greece (Rhea study). Int J Epidemiol 2017; 46: 1392-1393k.

20 Porta D, Fantini M. Prospective cohort studies of newborns in Italy to evaluate the role of environmental and genetic characteristics on common childhood disorders. Ital J Pediatr 2006; 32: 350.

21 Inskip HM, Godfrey KM, Robinson SM, et al. Cohort profile: the Southampton Women's Survey. Int J Epidemiol 2006; 35: 42-48.

22 Asher MI, Keil U, Anderson HR, et al. International study of asthma and allergies in childhood (ISAAC): rationale and methods. Eur Respir J 1995; 8: 483-491.

23 Deliu M, Belgrave D, Sperrin M, et al. Asthma phenotypes in childhood. Expert Rev Clin Immunol 2017; 13: $705-713$.

24 Martinez FD, Wright AL, Taussig LM, et al. Asthma and wheezing in the first six years of life. $N$ Engl J Med 1995; 332: $133-138$.

25 Cole TJ, Lobstein T. Extended international (IOTF) body mass index cut-offs for thinness, overweight and obesity. Pediatr Obes 2012; 7: 284-294.

26 De Onis M, Onyango AW, Borghi E, et al. Development of a WHO growth reference for school-aged children and adolescents. Bull World Health Organisation 2007; 85: 812-819.

27 WHO Multicentre Growth Reference Study Group. WHO child growth standards based on length/height, weight and age. Acta Paediatr Suppl 2006; 450: 76-85.

28 Berner A, Bornehag C. Breastfeeding less than three months increases the risk for airway and rhinitis symptoms in children. J Allergy Clin Immunol 2004; 113: S274-S275.

29 United Nations Children's Fund and World Health Organization. Low birthweight: country, regional and global estimates. New York, UNICEF, 2004. http://apps.who.int/iris/bitstream/handle/10665/43184/9280638327.pdf? sequence $=1$ \&isAllowed $=\mathrm{y}$

30 Clark TS, Linzer DA. Should I use fixed or random effects? Political Science Research and Methods 2015; 3: 399-408.

31 Textor J, Hardt J, Knüppel S. DAGitty: a graphical tool for analyzing causal diagrams. Epidemiology 2011; 22: $745-745$.

32 Subbarao P, Mandhane PJ, Sears MR. Asthma: epidemiology, etiology and risk factors. CMAJ 2009; 181, E181-E190.

33 Ly NP, Gold DR, Weiss ST, et al. Recurrent wheeze in early childhood and asthma among children at risk for atopy. Pediatrics 2006; 117: e1132-e1138.

34 Dixon AE, Poynter ME. Mechanisms of asthma in obesity: pleiotropic aspects of obesity produce distinct asthma phenotypes. Am J Respir Cell Mol Biol 2016; 54: 601-608.

35 Holguin F. Obesity as a risk factor for increased asthma severity and allergic inflammation; cause or effect? Clin Exp Allergy 2012; 42: 612-613.

36 Ahangari F, Sood A, Ma B, et al. Chitinase 3-like-1 regulates both visceral fat accumulation and asthma-like Th2 inflammation. Am J Respir Crit Care Med 2015; 191: 746-757.

37 Litonjua AA, Gold DR. Asthma and obesity: common early-life influences in the inception of disease. J Allergy Clin Immunol 2008; 121: 1075-1084.

38 Ford ES. The epidemiology of obesity and asthma. J Allergy Clin Immunol 2005; 115: 897-909.

39 Duijts L. Growing large and fast: is infant growth relevant for the early origins of childhood asthma? Thorax 2016; 71: 1071-1072.

40 Jani M, Ogston S, Mukhopadhyay S. Annual increase in body mass index in children with asthma on higher doses of inhaled steroids. J Pediatr 2005; 147: 549-551. 
41 Han J, Nguyen J, Kim Y, et al. Effect of inhaled corticosteroid use on weight (BMI) in pediatric patients with moderate-severe asthma. J Asthma 2018; in press [https://doi.org/10.1080/02770903.2018.1455853].

42 Monasta L, Lobstein T, Cole TJ, et al. Defining overweight and obesity in pre-school children: IOTF reference or WHO standard? Obes Rev 2011; 12: 295-300.

43 Zugna D, Galassi C, Annesi-Maesano I, et al. Maternal complications in pregnancy and wheezing in early childhood: a pooled analysis of 14 birth cohorts. Int J Epidemiol 2015; 44: 199-208.

44 Uphoff EP, Bird PK, Antó JM, et al. Variations in the prevalence of childhood asthma and wheeze in MeDALL cohorts in Europe. ERJ Open Res 2017; 3: 00150-2016.

45 Janssen I, Katzmarzyk PT, Boyce WF, et al. Comparison of overweight and obesity prevalence in school-aged youth from 34 countries and their relationships with physical activity and dietary patterns. Obes Rev 2005; 6 : 123-132.

46 World Health Organization Regional Office for Europe. Childhood Obesity Surveillance Initiative factsheet. Highlights 2015-2017. Preliminary data. Geneva, World Health Organization, 2018 www.euro.who.int/_data/ assets/pdf_file/0006/372426/wh14-cosi-factsheets-eng.pdf?ua=1 